\section{P177 WORK PARTICIPATION IN PATIENTS WITH SYSTEMATIC LUPUS ERYTHEMATOSUS: A SCOPING REVIEW}

${ }^{1}$ Birgit Blomjous, ${ }^{2}$ Gayle Gajadin, 'Irene Bultink, 'Alexandre Voskuyl, ${ }^{3}$ Louise Falzon, ${ }^{4}$ Jan Hoving, ${ }^{1,}{ }^{2}$ Marieke ter Wee. ${ }^{1}$ Dept. of Rheumatology, Amsterdam Rheumatology and immunology Center, Amsterdam Infection and Immunity, Amsterdam UMC, Vrije Universiteit Amsterdam, Amsterdam; ${ }^{2}$ Dept. of Epidemiology and Biostatistics, Amsterdam Public Health, Amsterdam UMC, Vrije Universiteit Amsterdam, Amsterdam, The Netherlands; ${ }^{3}$ Center for Personalized Health, Feinstein Institutes for Medical Research, Northwell Health, New York, USA; ${ }^{4}$ Coronel Institute of Occupational Health, Amsterdam Public Health Research Institute, Amsterdam UMC, University of Amsterdam, Amsterdam, The Netherlands

\subsection{6/lupus-2020-eurolupus.219}

Background The treatment of Systemic Lupus Erythematosus (SLE) has improved over the last decades. However, patients are more vulnerable for sick leave, work disability and unemployment compared to the general population. It is therefore important to understand the magnitude of the problem and the factors that might affect work participation in patients with SLE, to evaluate which actions can be taken to limit the burden on a patient and society level. The aim of this research is to summarize the existing literature on 1) the prevalence of work disability, 2) prognostic factors for work participation and 3 ) the effectiveness of interventions to improve work participation in patients with SLE.

Methods A scoping review, using the PRISMA-ScR statement will be conducted. Full-text original articles published before April 2019 were identified by literature search performed in MEDLINE, Cochrane, Embase and CINAHL. Articles in all languages, concerning work participation in patients with SLE, aged $\geq 18$ years were included.

Results In total, 2050 non-duplicate citations were screened. After title/abstract screening, 75 articles were retrieved. Relevant articles will be identified and reference lists will be searched for additional articles by two independent researchers. Information on author(s), year of publication, study location, intervention type, comparator, duration of the intervention, study population, aims of the study, methodology, outcome measures and important results will be collected. The results will be presented at the congress.

Conclusion Based on this scoping review, implications for future research including a research agenda and daily practice will be identified.

\section{P178 LUPUS NEPHRITIS WAS ASSOCIATED WITH LOW BONE MINERAL DENSITY IRRESPECTIVE OF DURATION ON STEROID TREATMENT IN A LARGE UK STUDY OF JUVENILE SYSTEMIC LUPUS ERYTHEMATOSUS}

${ }^{1}$ Anastasia-Vasiliki Madenidou, 1,2 Yasmin Mahfouz, 1,2 Oliver Chang, 1,2 Farah El-Sharnouby, ${ }^{1}$ Charlene Foley, ${ }^{1}$ Coziana Ciurtin. ${ }^{1}$ Centre for Adolescent Rheumatology, University College London, London; ' University College London Medical School, London, UK

\subsection{6/lupus-2020-eurolupus.220}

Background Estimated 10-20\% of all patients with systemic lupus erythematosus (SLE) develop clinical disease before the age of 18 years and are therefore classified as juvenile-onset SLE (JSLE). JSLE patients have a higher prevalence of lupus nephritis (LN) compared to adult-onset $\mathrm{SLE}^{1}$ and decreased long-term survival compared to JSLE patients without $\mathrm{LN}^{2}$ We aimed to identify clinical and laboratory predictors of $\mathrm{LN}$ in JSLE patients by comparing the baseline characteristics of JSLE patients with and without LN.

Methods This is a single-center retrospective study, who included JSLE patients reviewed in our young adult and adolescent clinics after transitioning from paediatric services. All data have been analyzed descriptively. Mann- Whitney U or Chi- square test were performed to compare the characteristics between the patients with and without LN. In addition, we performed uni and multivariate analysis to investigate potential predictor biomarkers for LN.

Results We identified 134 JSLE patients, out of which 45 (34\%) had LN. The baseline characteristics are detailed in table 1. As expected, the highest dsDNA levels ever were found in patients with $\mathrm{LN}(\mathrm{p}=0.022)$. Patients with $\mathrm{LN}$ also had longer disease duration $(p=0.012)$. The overall clinical manifestations and complications did not differ between JSLE patients with or without $\mathrm{LN}$, except for the prevalence of low

Abstract P178 Table 1 Baseline characteristics

\begin{tabular}{llll}
\hline & Lupus nephritis & Without Lupus nephritis & P value \\
\hline Number of patients, n (\%) & $45(36 \%)$ & $89(66.4 \%)$ & \\
Female & $39(86.7 \%)$ & $73(82 \%)$ & 0.624 \\
Age, years & $22(21-26)$ & $21(19-25)$ & 0.092 \\
Age at diagnosis, years & $13(10-15)$ & $13(11.25-15)$ & 0.471 \\
Age at menarche, years & $12.75(11.25-15.75)$ & $12.25(11-14.38)$ & 0.543 \\
Disease duration, years & $10(8-14)$ & $8(6-13)$ & $0.012^{*}$ \\
BMI, kg/m2 & $24.31(20.41-26.13)$ & $23.96(21.69-28.5)$ & 0.726 \\
ANA & $38(84.4 \%)$ & $85(95.5 \%)$ & 0.064 \\
ENA & $24(53.3 \%)$ & $51(57.3 \%)$ & 0.114 \\
Anti- Ro & $15(33.3 \%)$ & $31(34.8 \%)$ & 0.134 \\
Anti- La & $6(13.3 \%)$ & $14(15.7 \%)$ & 0.130 \\
RF & $3(6.7 \%)$ & $18(20.2 \%)$ & 0.114 \\
Highest dsDNA levels ever & $197(32-434)$ & $48(10-294)$ & $0.022^{*}$ \\
\hline
\end{tabular}

Numbers are medians (interquartile ranges) unless otherwise stated. * $p<0.05$ is significant

Abstract P178 Table 2 Clinical manifestations and complications

\begin{tabular}{llll}
\hline & Lupus nephritis & $\begin{array}{l}\text { Without Lupus } \\
\text { nephritis }\end{array}$ & P value \\
\hline Number of patients, $\mathrm{n}(\%)$ & $45(36 \%)$ & $89(66.4 \%)$ & \\
SLICC at last clinical assessment & 0 & $0(0-0.5)$ & 0.872 \\
SLEDAI at last clinical assessment & $2(0-4)$ & $0(0-2)$ & 0.123 \\
BILAG at last clinical assessment & $1(0-1.75)$ & $1(0-1)$ & 0.822 \\
Constitutional symptoms & $26(80 \%)$ & $67(75.3 \%)$ & 0.666 \\
Mouth ulcers & $31(68.8 \%)$ & $61(68.5 \%)$ & 0.258 \\
Skin involvement & $40(88.9 \%)$ & $73(82 \%)$ & 0.150 \\
Arthritis & $37(82.2 \%)$ & $71(79.8 \%)$ & 0.309 \\
Myositis & $5(11.1 \%)$ & $16(18 \%)$ & 0.451 \\
Neuropsychiatric involvement & $15(33.3 \%)$ & $19(21.3 \%)$ & 0.146 \\
Cardiorespiratory involvement & $12(26.7 \%)$ & $20(22.5 \%)$ & 0.669 \\
Gastrointestinal involvement & $8(17.8 \%)$ & $18(20.2 \%)$ & 0.724 \\
Ophthalmic involvement & $4(8.9 \%)$ & $9(10.1 \%)$ & 0.108 \\
Haematological involvement & $26(57.8 \%)$ & $44(49.4 \%)$ & 0.464 \\
Hypertension & $8(17.8 \%)$ & $6(6.7 \%)$ & 0.071 \\
Stroke & $1(2.2 \%)$ & $2(2.2 \%)$ & 1.000 \\
Low bone mineral density & $16(35.5 \%)$ & $11(12.4 \%)$ & $0.008 *$ \\
Malignancy & $1(2.2 \%)$ & $1(1.1 \%)$ & 1.000 \\
\hline Numbers are medians (interquartile ranges) & unless otherwise stated. ${ }^{*} \mathrm{p}<0.05$ is significant
\end{tabular}




\begin{tabular}{llll}
$\begin{array}{l}\text { Abstract P178 Table 3 } \\
\text { patients }\end{array}$ & Treatment differences among JSLE & \\
\hline & $\begin{array}{l}\text { Lupus } \\
\text { nephritis }\end{array}$ & $\begin{array}{l}\text { Without Lupus } \\
\text { nephritis }\end{array}$ & p \\
\hline Number of patients, n (\%) & $45(36 \%)$ & $89(66.4 \%)$ & \\
Hydroxychloroquine, n (\%) & $43(95.6 \%)$ & $88(98.9 \%)$ & 0.295 \\
Steroid treatment duration, (months) & $31(1.5-84.5)$ & $29.5(9-54.8)$ & 0.834 \\
MMF, n (\%) & $39(86.7 \%)$ & $43(48.3 \%)$ & $0.000^{*}$ \\
MMF, months & $42(18.75-94.5)$ & $0(0-44)$ & $0.000^{*}$ \\
Cyclophosphamide, n (\%) & $22(48.9 \%)$ & $17(19.1 \%)$ & $0.001^{*}$ \\
Cyclophosphamide, number of courses & $0(0-3)$ & 0 & $0.000^{*}$ \\
Rituximab, n (\%) & $27(60 \%)$ & $29(32.6 \%)$ & $0.003^{*}$ \\
Rituximab, number of courses & $2(0-2)$ & $0(0-1)$ & $0.000^{*}$ \\
Azathioprine, months & $2.5(0-48.5)$ & $10(0-41)$ & 0.868 \\
Methotrexate, months & 0 & $0(0-0.75)$ & 0.529 \\
Tacrolimus, months & 0 & 0 & 0.219 \\
Belimumab, months & 0 & 0 & 0.310 \\
\hline
\end{tabular}

Numbers are medians (interquartile ranges) unless otherwise stated. MMF= mycophenolate mofetil. * $p<0.05$ is significant

bone mineral density (table 2). Factors associated with low bone mineral density in a univariate logistic regression analysis were: the use of mycophenolate mofetil $(p=0.021)$ and the presence of $\mathrm{LN}(\mathrm{p}=0.007)$, while $\mathrm{LN}$ also associated with low bone density $(p=0.008)$ in a multivariate analysis. The skin involvement was the most common clinical manifestation in both JSLE patient groups. The treatment was more aggressive in patients with $\mathrm{LN}$, including the use of cyclophosphamide, mycophenolate and rituximab, but there was no difference regarding the length of the steroid treatment (table 3 ). The majority of the patients $(67 \%)$ had one flare of nephritis and the number of flares ranged from $2(13 \%)$ to $12(2.2 \%)$ for the rest of the patients in this cohort with disease duration ranging from $8-12$ years. The class of nephritis was reported in 34 out of 45 patients, and focal lupus nephritis (class III) was the most common type (44\%). There were no statistical significant differences in the baseline characteristics or treatments among the different classes of LN.

Conclusion Low bone mineral density is common in patients with $\mathrm{JSLE}^{3}$, but this is the first study highlighting the association of low bone density with LN and the lack of association with the total duration of steroid treatment or other clinical manifestations.

\section{REFERENCES}

1. Ambrose $\mathrm{N}$, et al. Differences in disease phenotype and severity in SLE across age groups. Lupus. 2016;25(14):1542-50.

2. Smith EMD, et al. Clinical predictors of active LN development in children- evidence from the UK JSLE Cohort Study.

3. Compeyrot-Lacassagne $S$, et al. Prevalence and etiology of low bone mineral density in Juvenile Systemic Lupus Erythematosus.

\section{\begin{tabular}{|l|l}
\hline P179 SYSTEMIC LUPUS ERYTHEMATOSUS, A PILOT STUDY OF \\
\hline
\end{tabular} A NEW DISEASE ACTIVITY SCORE}

${ }^{1}$ Daphna Paran, ${ }^{1}$ Monique Ben-Am, 'Liran Mendel, ${ }^{1}$ Ari Polachek, ${ }^{1}$ Victoria Furer, ${ }^{1}$ Ofir Elalouf, ${ }^{1}$ Jonathan Wollman, ${ }^{2}$ Shaye Kivity, ${ }^{3}$ Nancy Agmon-Levin. ${ }^{1}$ Dept. of Rheumatology, Tel Aviv Medical Center; 'Dept. of Medicine 'A', Sheba Medical Center; ${ }^{3}$ Clinical Immunology, Angioedema and Allergy Unit, Center for Autoimmune Diseases, Sheba Medical Center and Tel-Aviv University, Israel

10.1136/lupus-2020-eurolupus.221
Background SLE complexity and unpredictability challenge assessment of disease activity. Current scoring instruments are limited in ability to detect changes in activity over time and too cumbersome for daily practice. We constructed a new disease activity score, including physician and patient assessment, aiming to simplify and improve assessment in daily practice, and possibly serve as a tool for clinical studies.

Methods The new instrument is comprised of 6 visual analogue scales, separately addressing the physician's global assessment and 5 organ systems: mucocutaneous, musculoskeletal, cardiorespiratory, renal and neuropsychiatric systems, and 5 visual analogue scales addressing patients' assessment of disease activity and adherence to therapy. Laboratory values and medications are recorded. Aiming to assess the reliability and validity of the new score, as well as it's sensitivity to changes in disease activity, 4 paper cases, including 2 visits per case, were constructed. Each visit was scored by 5 experienced rheumatologists, using BILAG, SLEDAI, LFA-REAL and our proposed score.

Results The inter-rater reliability of the new score was good for all systems, both for single visit scores and for change in disease activity between 2 consecutive visits (ICC $[2,1]$ range $0.75-0.95)$, except for changes in activity in the renal system $(0.59)$. The inter-rater reliability values of the new score were comparable with those of the BILAG and the LFA-REAL instruments. The construct validity of the new score was good for single visit scores (Spearman correlation coefficients range 0.48-0.94). Correlation of our proposed score with the BILAG, was good when scoring the mucocutaneous, musculoskeletal and cardiorespiratory systems $(0.66,0.75,0.83$, respectively) but poor when scoring the renal system $(0.11)$.

Conclusions This paper case evaluation of the new disease activity score suggests a promising and simple tool, with overall good reliability and construct validity.

\section{P180 CORRELATION BETWEEN PATIENT REPORTED OUTCOMES OF HEALTH-RELATED QUALITY OF LIFE AND CLINICAL ACTIVITY IN SYSTEMIC LUPUS ERYTHEMATOSUS PATIENTS}

${ }^{1}$ Elena Grau-García, 'Samuel Leal-Rodríguez, ${ }^{1}$ Cristóbal Pávez-Perales, ${ }^{1}$ Cristina AlcañizEscandell, 'Inés Cánovas-Olmos, ${ }^{1}$ Inmaculada Chalmeta-Verdejo, ${ }^{1}$ Marta De-la-RubiaNavarro, 'Jorge Juan Fragio-Gil, 'Roxana González-Mazarío, 'Luis González-Puig, 'José Ivorra-Cortés, ${ }^{1}$ Isabel Martínez-Cordellat, ${ }^{1}$ Carmen Nájera-Herranz, ${ }^{1}$ Rosa NeguerolesAlbuixech, 'José Eloy Oller-Rodríguez, ${ }^{1}$ Francisco Miguel Ortiz-Sanjuán, 'Elvira VicensBernabeu, ${ }^{2}$ Daniel Hervás-Marín, ${ }^{1} J o s e ́$ Andrés Román-Ivorra. ${ }^{1}$ Rheumatology Dept., Hospital Universitario y Politécnico de La Fe, Valencia; ${ }^{2}$ Biostatistics Unit. Instituto de Investigación Sanitaria La Fe, Valencia, Spain

\subsection{6/lupus-2020-eurolupus.222}

Background/Purpose Patient-Reported Outcomes (PROs) allow us to know how the disease could affect patients, and maybe could not be detected by clinical measures. Among these, PROs of health-related quality of life (PRO-QL) represents patient evaluation of its health status. In systemic lupus erythematosus (SLE) patients, the heterogeneous inflammatory symptoms can affect the health-related quality of life in different ways.

We aimed to measure the PRO-QL in SLE patients and correlate them with the clinical activity of the disease.

Methods A cross-sectional observational study with SLE patients diagnosed according to SLICC 2012 criteria was performed. SLEDAI score was carried out, and patients full-filled 